\title{
Endoscopic third ventriculostomy with choroid plexus cauterization outcome: distinguishing success from failure
}

\author{
Michael C. Dewan, MD, Jaims Lim, BS, Clinton D. Morgan, MD, Stephen R. Gannon, BS, \\ Chevis N. Shannon, MBA, MPH, DrPH, John C. Wellons III, MD, MSPH, and Robert P. Naftel, MD \\ Department of Neurosurgery, Vanderbilt University, Division of Pediatric Neurosurgery, Monroe Carell Jr. Children's Hospital at \\ Vanderbilt, Nashville, Tennessee
}

\begin{abstract}
OBJECTIVE Endoscopic third ventriculostomy with choroid plexus cauterization (ETV/CPC) offers an alternative to shunt treatment for infantile hydrocephalus. Diagnosing treatment failure is dependent on infantile hydrocephalus metrics, including head circumference, fontanel quality, and ventricle size. However, it is not clear to what degree these metrics should be expected to change after ETVICPC. Using these clinical metrics, the authors present and analyze the decision making in cases of ETV/CPC failure.
\end{abstract}

METHODS Infantile hydrocephalus metrics, including bulging fontanel, head circumference z-score, and frontal and occipital horn ratio (FOHR), were compared between ETV/CPC failures and successes. Treatment outcome predictive values of metrics individually and in combination were calculated.

RESULTS Forty-four patients ( $57 \%$ males, median age 1.2 months) underwent ETV/CPC for hydrocephalus; of these patients, $25(57 \%)$ experienced failure at a median time of 51 days postoperatively. Patients experiencing failure were younger than those experiencing successful treatment ( 0.8 vs 3.9 months, $p=0.01$ ). During outpatient follow-up, bulging anterior fontanel, progressive macrocephaly, and enlarging ventricles each demonstrated a positive predictive value (PPV) of no less than $71 \%$, but a bulging anterior fontanel remained the most predictive indicator of ETV/CPC failure, with a PPV of $100 \%$, negative predictive value of $73 \%$, and sensitivity of $72 \%$. The highest PPVs and specificities existed when the clinical metrics were present in combination, although sensitivities decreased expectedly. Only $48 \%$ of failures were diagnosed on the basis all 3 hydrocephalus metrics, while only $37 \%$ of successes were negative for all 3 metrics. In the remaining $57 \%$ of patients, a diagnosis of success or failure was made in the presence of discordant data.

CONCLUSIONS Successful ETVICPC for infantile hydrocephalus was evaluated in relation to fontanel status, head growth, and change in ventricular size. In most patients, a designation of failure or success was made in the setting of discordant data.

http://thejns.org/doi/abs/10.3171/2016.6.PEDS1675

KEY WORDS choroid plexus cauterization; endoscopic third ventriculostomy; frontooccipital horn ratio; hydrocephalus

$\mathrm{E}$ NDOSCOPIC third ventriculostomy with choroid plexus cauterization (ETV/CPC) for the treatment of infantile hydrocephalus is being performed as an alternative to shunting. ${ }^{7,11,12,16}$ Multiple validated success scales exist as simple tools to calculate the expected success rate of endoscopically treated hydrocephalus based on preoperative variables. ${ }^{6,15}$ All of these predictive models depend on the manner in which the diagnosis of failure is established. The decision making in diagnosing ETV/
CPC success or failure remains subjective, ambiguous, and often opaque in current studies.

Metrics of assessing infantile hydrocephalus include head circumference, ventricular size, and fontanel quality. In traditional treatment of infantile hydrocephalus, a ventriculoperitoneal shunt is inserted, and it is expected that all 3 of these metrics will begin to normalize or "improve," i.e., the fontanel will flatten and soften, accelerated head growth will cease, and ventricle size will decrease. In

ABBREVIATIONS ETVICPC = endoscopic third ventriculostomy with choroid plexus cauterization; FOHR = frontal and occipital horn ratio; ICP = intracranial pressure; IQR $=$ interquartile range; $\mathrm{IVH}=$ intraventricular hemorrhage.

SUBMITTED February 8, 2016. ACCEPTED June 17, 2016.

INCLUDE WHEN CITING Published online August 26, 2016; DOI: 10.3171/2016.6.PEDS1675. 
previous ETV/CPC series, failure has been defined when additional surgery is needed to treat hydrocephalus. ${ }^{11-14,16}$ These studies reported using accelerated head circumference growth rate, bulging anterior fontanel, and progressive ventriculomegaly as clinical factors used in diagnosing failure. ${ }^{2,11,13}$ What remains unclear is to what degree each criterion individually contributes to a designation of treatment failure. Therefore, this study examines decision making in ETV/CPC failure by analyzing postoperative metrics used to diagnose failure.

\section{Methods}

\section{Design and Study Population}

This was a retrospective study examining postoperative clinical metrics between patients experiencing ETV/ CPC failure or success. Between 2013 and 2015, patients undergoing ETV/CPC for treatment of hydrocephalus were prospectively enrolled in an institutional pediatric hydrocephalus registry. Relevant demographic, clinical, and radiographic data were recorded from presentation until the last clinical follow-up (minimum 6 months). At our institution the surgical procedure was performed by one of 2 surgeons using a flexible endoscope. This procedure has been previously described for treating infants with hydrocephalus. ${ }^{12}$ Institutional review board approval was obtained for this study.

\section{Clinical Metrics for ETV/CPC Outcome}

Traditionally, ETV/CPC success is designated by the absence of either a second treatment for hydrocephalus or death due to hydrocephalus. ${ }^{6,12}$ Determination of failure is commonly attributed to any combination of the following: progressive ventriculomegaly, bulging anterior fontanel, abnormally progressive head circumference growth, and worsening signs or symptoms of elevated intracranial pressure (ICP). ${ }^{12}$ Given the very young age of our population, subjective variables, including symptoms of elevated ICP, were not included in our analysis in an effort to avoid ambiguity.

Head circumference was obtained in the standard fashion at the bedside using a disposable tape measure. ${ }^{1}$ All measurements were performed by a nurse and were then confirmed by a physician. Any discrepancy in measurement resulted in repeat measurement by the physician. The head circumference $\mathrm{z}$-score was calculated from the head circumference measurement in centimeters, as described by the 2006 WHO Child Growth Standards (http://www. who.int/childgrowth/en/). Briefly, the head circumference $\mathrm{z}$-score is the deviation of the head circumference value for an individual from the mean value of the reference population divided by the standard deviation for the reference population. The z-score can be corrected for age group and is an attractive, accepted alternative to simply documenting head circumference percentiles, as it provides a single directional unit reflecting head circumference deviation. The z-scores and corresponding head circumference percentile can be easily calculated using a standardized chart or formula available on the Internet (http://www.uptodate. $\mathrm{com} /$ contents/calculator-who-infant-head-circumferencefor-age-percentiles-less-than24-months). Using the head circumference z-score rather than head circumference percentiles has the advantage of normalizing the values, allowing more useful comparisons at the extremes of distribution (where many hydrocephalus metrics reside). As a reference, a head circumference z-score of -1 roughly correlates with the 15th percentile, while a z-score of 1 approaches the 85 th percentile. Similarly, a z-score of -2 equals the 2.3 percentile, while a score of 2 approaches the 98th percentile.

Ventricle size was measured by trained members of the research team using the frontal and occipital horn ratio (FOHR), which is a validated ratio representing ventricular volume and is found to have high interobserver reliability in pediatric patients. ${ }^{9}$ Preoperative FOHR was measured using MRI, while MRI, ultrasonography, and CT were used with variability to measure postoperative FOHR, and depended on the follow-up study performed at the designated visit. While a uniform imaging modality was not used in this study, the interchangeability of several modalities has been previously described. ${ }^{9}$ Fontanel quality was assessed by neurosurgical clinicians and documented in the medical record. Fontanel quality was defined using binary values: a bulging fontanel was defined as above the level of the surrounding external table of bone. Absence of a bulging fontanel was denoted when the fontanel was palpated at or below the level of the surrounding external table of bone (flat or concave). This definition has been found to be highly reliable. ${ }^{17}$

Postoperatively, patients underwent clinical evaluation at 2 weeks, 6 weeks, 3 months, 6 months, 9 months, and 12 months, or at any time in between if interim neurosurgical evaluation was clinically necessary. Cranial imaging in the form of a fast-sequence brain MRI study, cranial ultrasound, or rarely, head CT scanning was performed in all patients at the 2-week, 6-week, 3-month, and 6-month appointments and at any time in between if there was concern for progressive hydrocephalus. The final clinical and radiographic parameters used for analysis among successes were those taken at the 6-month follow-up visit, while parameters for failures were those documented during the clinical visit at which failure was designated.

First, after correction of age for prematurity, preoperative variables, including age, fontanel quality, head circumference, and ventricular size, were compared between the 2 groups to assess pretreatment variability. Next, from the postoperative period, the same hydrocephalus metrics were compared between ETV failures and successes. Additionally, the changes (Value preoperative - Value 6-month/failure $_{\text {re }}$ in head circumference, head circumference percentile ( $\Delta \%$ ile), head circumference z-score $(\Delta \mathrm{z}$-score), and FOHR $(\Delta \mathrm{FOHR})$ were also documented and compared between groups. A positive $\Delta z$-score indicated an increase in the head circumference that was deviating further from the mean than the growth curve would project. A positive $\triangle F O H R$ indicates an increase in the size of the ventricles. Naturally, a negative value of each $\Delta$ value represents the opposite.

\section{Statistical Analysis}

Study data were collected and managed using Research Electronic Data Capture (REDCap) tools. ${ }^{3}$ Data analysis 
TABLE 1. Baseline characteristics of 44 infants with hydrocephalus treated with ETV/CPC

\begin{tabular}{lc}
\hline \multicolumn{1}{c}{ Characteristics } & Value* \\
\hline Male sex & $25(57)$ \\
\hline Age (mos) & $3.9 \pm 5.3$ \\
\hline Mean \pm SD & $1.2[0.6-5.6]$ \\
\hline Median [IQR] & $36(82)$ \\
\hline Signs/symptoms & $40(91)$ \\
\hline Increasing HC & $4(9)$ \\
\hline Bulging fontanel & $2(5)$ \\
\hline Ocular palsy/paresis & \\
\hline Apneic/bradycardic episodes & $20(46)$ \\
\hline Etiology of hydrocephalus & $9(20)$ \\
\hline Myelomeningocele & $9(20)$ \\
\hline IVH & $3(7)$ \\
\hline Aqueductal stenosis & $3(7)$ \\
\hline CCH & $6(14)$ \\
\hline Other & $3(7)$ \\
\hline History or prior intervention for hydrocephalus & $3(7)$ \\
\hline Subgaleal shunt &
\end{tabular}

$\mathrm{CCH}=$ congenital communicating hydrocephalus; $\mathrm{HC}=$ head circumference.

* Values are presented as the number of patients (\%) unless indicated otherwise.

† Craniosynostosis (1, success), Dandy-Walker cyst (1, failure), and other intracranial cyst (1, success).

was carried out in a de-identified manner by the primary and senior authors using IBM SPSS software (version 23).

Data were summarized using counts and frequencies for categorical variables with relative proportions as percentages. Descriptive data were given as the median with interquartile range (IQR) or mean with standard deviation (SD) where appropriate. Continuous, nonnormally distrib- uted data were compared using the Mann-Whitney U-test. Dichotomous data were compared between the 2 groups by using the chi-square test, and comparisons of binary variables with fields containing fewer than 5 frequencies were compared using the Fisher's exact test. Positive and negative predictive values as well as sensitivity/specificity were calculated using standard 2-by-2 table methodology.

\section{Results}

\section{Patient Characteristics}

Forty-four patients (25 male, mean/median age 3.9/1.2 months) underwent ETV/CPC for hydrocephalus (Table 1). Patients developed hydrocephalus secondary to myelomeningocele $(20,46 \%)$, intraventricular hemorrhage (IVH) $(9,20 \%)$, aqueductal stenosis $(9,20 \%)$, congenital communicating hydrocephalus $(3,7 \%)$, and other causes $(3,7 \%)$. On presentation, 36 patients (82\%) had progressively increasing head circumference, $40(91 \%)$ had a bulging fontanel, and $4(9 \%)$ had ocular palsy related to hydrocephalus. Six (14\%) patients underwent previous hydrocephalus treatments, including subgaleal and ventriculoperitoneal shunts before ETV/CPC. Among the 44-patient cohort, at presentation, the median head circumference percentile and z-score were 98\% (IQR 89\%-99\%) and 2.2 (IQR 1.2-4.4), respectively. All patients had ventriculomegaly with a median FOHR of 0.53 (IQR 0.48-0.59).

\section{ETV/CPC Failures}

Twenty-five patients (57\%) experienced ETV/CPC failure and required additional intervention for treatment of hydrocephalus (Table 2). The mean and median times to failure were 80 and 51 days, respectively. Relative to successes, patients in whom ETV/CPC failed underwent surgery at a younger age (median age 0.8 vs 3.9 months, $\mathrm{p}=0.01$ ). Furthermore, there was a positive correlation between the age at index surgery and time to failure, wherein younger patients tended to experience failure sooner than

TABLE 2. Baseline comparison of preoperative variables between patients in the ETV/CPC failures and successes

\begin{tabular}{lccc}
\hline & \multicolumn{2}{c}{ Failure } & p Value \\
\cline { 2 - 3 } Variable & Yes $(\mathrm{n}=25)$ & No $(\mathrm{n}=19)$ & 0.010 \\
\hline Median age [IQR], mos & $0.82[0.5-2.0]$ & $3.9[1.0-9.4]$ & 1.0 \\
\hline Bulging fontanel & $92 \%(23 / 25)$ & $89 \%(17 / 19)$ & 0.008 \\
\hline $\mathrm{HC}$ & & & 0.187 \\
\hline Median [IQR], cm & $38.0[36.0-42.5]$ & $44.0[39.0-46.8]$ & 0.222 \\
\hline Median percentile [IQR] & $99[89-99]$ & $95[88-99]$ & 0.013 \\
\hline Median z-score [IQR] & $2.8[1.2-4.4]$ & $1.6[1.3-2.7]$ & 0.26 \\
\hline Median FOHR [IQR] & $0.56[0.50-0.59]$ & $0.51[0.48-0.54]$ & \\
\hline Etiology of hydrocephalus & & & $11(55 \%)$ \\
\hline Myelomeningocele $(n=20)$ & $9(45 \%)$ & $3(33 \%)$ & \\
\hline IVH $(n=9)$ & $6(67 \%)$ & $2(22 \%)$ & $1(33 \%)$ \\
\hline Aqueductal stenosis $(n=9)$ & $7(78 \%)$ & $2(66 \%)$ & \\
\hline CCH $(n=3)$ & $2(66 \%)$ & $1(33 \%)$ & \\
\hline Other $(n=3)^{*}$ & & & \\
\hline
\end{tabular}

* Craniosynostosis (1, success), Dandy-Walker cyst (1, failure), and other intracranial cyst (1, success). 
TABLE 3. Comparison of postoperative hydrocephalus metrics between patients experiencing ETV/CPC failures and successes

\begin{tabular}{|c|c|c|c|}
\hline \multirow[b]{2}{*}{ Variable } & \multicolumn{2}{|c|}{ Failure } & \multirow[b]{2}{*}{$p$ Value } \\
\hline & Yes $(n=25)$ & No $(n=19)$ & \\
\hline Bulging fontanel & $72 \%(18 / 25)$ & $0 \%(0 / 19)$ & $<0.001$ \\
\hline \multicolumn{4}{|l|}{$\mathrm{HC}$} \\
\hline $\begin{array}{l}\text { Median percentile } \\
\text { [IQR], \% }\end{array}$ & 99 [98-99] & 93 [70-99] & 0.001 \\
\hline $\begin{array}{l}\text { Mean percentile } \\
\text { (SD), \% }\end{array}$ & $5.4(11.3)$ & $-8.8(18.9)$ & 0.002 \\
\hline Median z-score [IQR] & $3.9[2.5-5.2]$ & $1.5[0.5-2.7]$ & $<0.001$ \\
\hline Mean $\Delta$ z-score (SD) & $0.96(1.3)$ & $-0.66(1.4)$ & $<0.001$ \\
\hline \multicolumn{4}{|l|}{ FOHR } \\
\hline Median [IQR] & 0.63 [0.58-0.69] & $0.48[0.47-0.53]$ & $<0.001$ \\
\hline Mean $\Delta \mathrm{FOHR}(\mathrm{SD})$ & $0.07(0.06)$ & $-0.01(0.07)$ & $<0.001$ \\
\hline
\end{tabular}

their older counterparts (Spearman's rho $=0.446, \mathrm{p}=$ $0.029)$. Colinear with age, the median preoperative head circumference was smaller in patients experiencing ETV/ CPC failure (38.0 vs $44.0 \mathrm{~cm}, \mathrm{p}=0.008$ ). Accounting for age difference and growth curves, the head circumference percentile and z-score did not differ between groups. The preoperative median FOHR was larger-albeit minimally-in the failure group than in the successful group ( 0.56 vs $0.51, p=0.013)$. Compendiously, the failures were predominantly younger patients who started with slightly larger ventricles. Preoperative presence or absence of a bulging fontanel and etiology of hydrocephalus were similar between the 2 groups (Table 2).

Next, postoperative hydrocephalus metrics were compared between the successes and failures. During followup from ETV/CPC, a bulging fontanel was present in $72 \%$ of patients experiencing failure and none of those experiencing successful ETV/CPC ( $<$ 0.001) (Table 3). On average, patients experiencing failure noted continued accelerated head circumference growth with a median head circumference percentile 6 points higher among the failure group (99\% vs $93 \%, \mathrm{p}=0.001$ ), and the median head circumference $\mathrm{z}$-score was 2.4 greater (3.9 vs 1.5 , $\mathrm{p}<0.001$ ). Relative to successes, patients experiencing failure exhibited increases from preoperative measures in both head circumference percentile $(5.4 \%$ vs $-8.8 \%, \mathrm{p}=$ $0.002)$ and $z$-score $(0.96$ vs $-0.66, \mathrm{p}<0.001)$. As expected, the median post-ETV/CPC FOHR was greater in patients experiencing failure $(0.63)$ than those achieving success $(0.48)(\mathrm{p}<0.001)$. Among patients who underwent a successful ETV/CPC, on average there was little change in FOHR from preoperatively $(-0.01)$, while failures demonstrated a small but significant increase of $0.07(\mathrm{p}<0.001)$ (Table 3). No patient (with failure or otherwise) presented in follow-up with hemodynamic changes that were consistent with elevated ICP (apnea and bradycardia); thus, these parameters were not considered within the decision model.

\section{Hydrocephalus Metrics Predictive Values}

Using these postoperative objective metrics, decision
TABLE 4. Diagnostic accuracy of hydrocephalus metrics for ETV/ CPC failure

\begin{tabular}{lrrr}
\hline \multicolumn{1}{c}{ Diagnostic Metric(s) } & \multicolumn{1}{c}{ PPV, $\%$} & NPV, \%* & p Value \\
\hline Bulging fontanel & $100(18 / 18)$ & $73(19 / 26)$ & $<0.001$ \\
\hline$\Delta$ FOHR $>0$ & $71(22 / 31)$ & $77(10 / 13)$ & 0.007 \\
\hline$\Delta H C$ z-score $>0$ & $83(19 / 23)$ & $71(15 / 21)$ & 0.001 \\
\hline Bulging fontanel + $\Delta$ FOHR $>0$ & $100(16 / 16)$ & $68(19 / 28)$ & $<0.001$ \\
\hline $\begin{array}{l}\text { Bulging fontanel + } \Delta \mathrm{HC} \text { z- } \\
\text { score }>0\end{array}$ & $100(14 / 14)$ & $63(19 / 30)$ & $<0.001$ \\
\hline $\begin{array}{l}\Delta H C \text { z-score }>0+\Delta \text { FOHR }>0 \\
\text { Bulging fontanel }+\Delta H C \text { z- } \\
\text { score }>0+\Delta \text { FOHR }>0\end{array}$ & $94(16 / 17)$ & $67(18 / 27)$ & $<0.001$ \\
\hline
\end{tabular}

* For calculation, combinations are considered a negative if any one factor was negative.

$\dagger p$ values represent strength of association between diagnostic metrics and occurrence of ETVICPC failure as tested via chi-square analysis in the same 2 $\times 2$ table used to calculate PPV and NPV.

making in ETV/CPC failure was then investigated. Necessarily, only postoperative characteristics were considered, as these variables constituted the clinical data by which the surgeon determined ETV/CPC failure. Fontanel quality, measures of head circumference (including absolute measure [cm], percentile, and z-score), and degree of ventriculomegaly (FOHR and $\triangle F O H R$ ) all carried significant value differences between ETV/CPC failure and success (Table 3). Using these 3 parameters, a simplified set of diagnostic combinations was developed to understand the contribution of each metric to the designation of failure.

The presence of a bulging fontanel was the most predictive indicator for ETV/CPC failure, with a positive predictive value (PPV) of $100 \%$ and negative predictive value (NPV) of $73 \%$, meaning that $100 \%$ of patients with a bulging fontanel were treated as experiencing ETV/CPC failure, and $73 \%$ of patients with a flat or concave fontanel did not experience failure (Table 4). The sensitivity and specificity of a bulging fontanel in the designation of ETV/ CPC failure were $72 \%$ and $100 \%$, respectively (Table 5). Essentially, the presence of a flat or concave fontanel renders a diagnosis of ETV/CPC failure unlikely, regardless of what imaging may reveal. The change in head circumference z-score and FOHR were also individually predictive of ETV/CPC outcome but not as strongly as fontanel status. During follow-up, a change in head circumference z-score $>0$ was $76 \%$ sensitive and $79 \%$ specific for failure and carried a PPV of $83 \%$ and NPV of $71 \%$. On the other hand, a follow-up $\triangle \mathrm{FOHR}>0$ was $88 \%$ sensitive but only $53 \%$ specific, with a PPV of $71 \%$ and NPV of $77 \%$. As would be expected, in combination, the PPV and specificities increase while the sensitivities decrease.

Finally, the predictive values were calculated for the scenario wherein 2 or more metrics were positive in any given patient (relative to 1 or 0 being positive). From among this cohort, the PPV was $95 \%$ (21/22), the NPV was $83 \%$ $(15 / 18)$, the sensitivity was $88 \%$ (21/24), and the specificity was $94 \%(15 / 16)$.

Importantly, a regression model was constructed, but care was taken not to include it in the paper for 2 reasons. First, covariance among the variables produced nonsensi- 
TABLE 5. Diagnostic accuracy of hydrocephalus metrics for ETV/ $\mathrm{CPC}$ failure

\begin{tabular}{|c|c|c|c|}
\hline Diagnostic Metric(s) & $\begin{array}{c}\text { Sensitivity, } \\
\%\end{array}$ & $\begin{array}{c}\text { Specificity, } \\
\%\end{array}$ & $\begin{array}{c}p \\
\text { Value* }^{*}\end{array}$ \\
\hline Bulging fontanel & $72(18 / 25)$ & $100(19 / 19)$ & $<0.001$ \\
\hline$\Delta \mathrm{FOR}>0$ & $88(22 / 25)$ & $53(10 / 19)$ & 0.007 \\
\hline$\Delta \mathrm{HC}$ z-score $>0$ & $76(19 / 25)$ & $79(15 / 19)$ & 0.001 \\
\hline Bulging fontanel $+\Delta \mathrm{FOR}>0$ & $64(16 / 25)$ & $100(19 / 19)$ & $<0.001$ \\
\hline Bulging fontanel $+\Delta \mathrm{HC}$ z-score $>0$ & $56(14 / 25)$ & $100(19 / 19)$ & $<0.001$ \\
\hline$\Delta \mathrm{HC}$ z-score $>0+\Delta \mathrm{FOHR}>0$ & $64(16 / 25)$ & $95(18 / 19)$ & $<0.001$ \\
\hline $\begin{array}{l}\text { Bulging fontanel }+\Delta \mathrm{HC} \text { z-score } \\
>0+\Delta \mathrm{FOHR}>0\end{array}$ & $48(12 / 25)$ & $100(19 / 19)$ & 0.62 \\
\hline
\end{tabular}

* $p$ values represent strength of association between diagnostic metrics and occurrence of ETVICPC failure as tested via chi-square analysis in the same 2 $\times 2$ table used to calculate sensitivity and specificity.

cal predictive coefficient output. Second, building a multivariate predictive model would risk invalid statistical conclusions because the dependent variable (ETV/CPC outcome) is the very entity that the independent variables are attempting to define.

\section{Decision Making}

Decision making is outlined in Fig. 1, which demonstrates interactions among all 3 hydrocephalus metrics and patient success or failure. Of the 25 failures, 12 infants presented with all 3 metrics indicating failure. Of the 19 successes, 7 infants presented with all metrics indicating success. For the remaining 25 patients, decisions of success and failure were made from discordant data. For failures, $88 \%$ were diagnosed with 2 or more hydrocephalus metrics indicating failure (areas of overlap on the Venn diagram) with the remaining 3 failures presenting with only 1 failure metric. If a bulging fontanel was present, it was always present with another hydrocephalus failure metric.

On the other hand, $12(63 \%)$ patients whose treatment was successful presented with at least 1 failure hydrocephalus metric. Only 1 patient whose treatment was successful had 2 failure metrics. Nearly half (47\%) of patients with successful treatment had larger ventricles and $21 \%$ had progressive macrocephaly in follow-up.

\section{Discussion}

In this study, decision making in ETV/CPC failure was investigated, and in the majority (57\%) of cases, failure or success was diagnosed using discordant data. Although the sample size is small, it is representative for ETV/CPC studies. Importantly, all patients were treated for symptomatic hydrocephalus, with $91 \%$ of patients having a bulging fontanel and $82 \%$ of patients presenting with progressive macrocephaly. Additionally, the success rate of

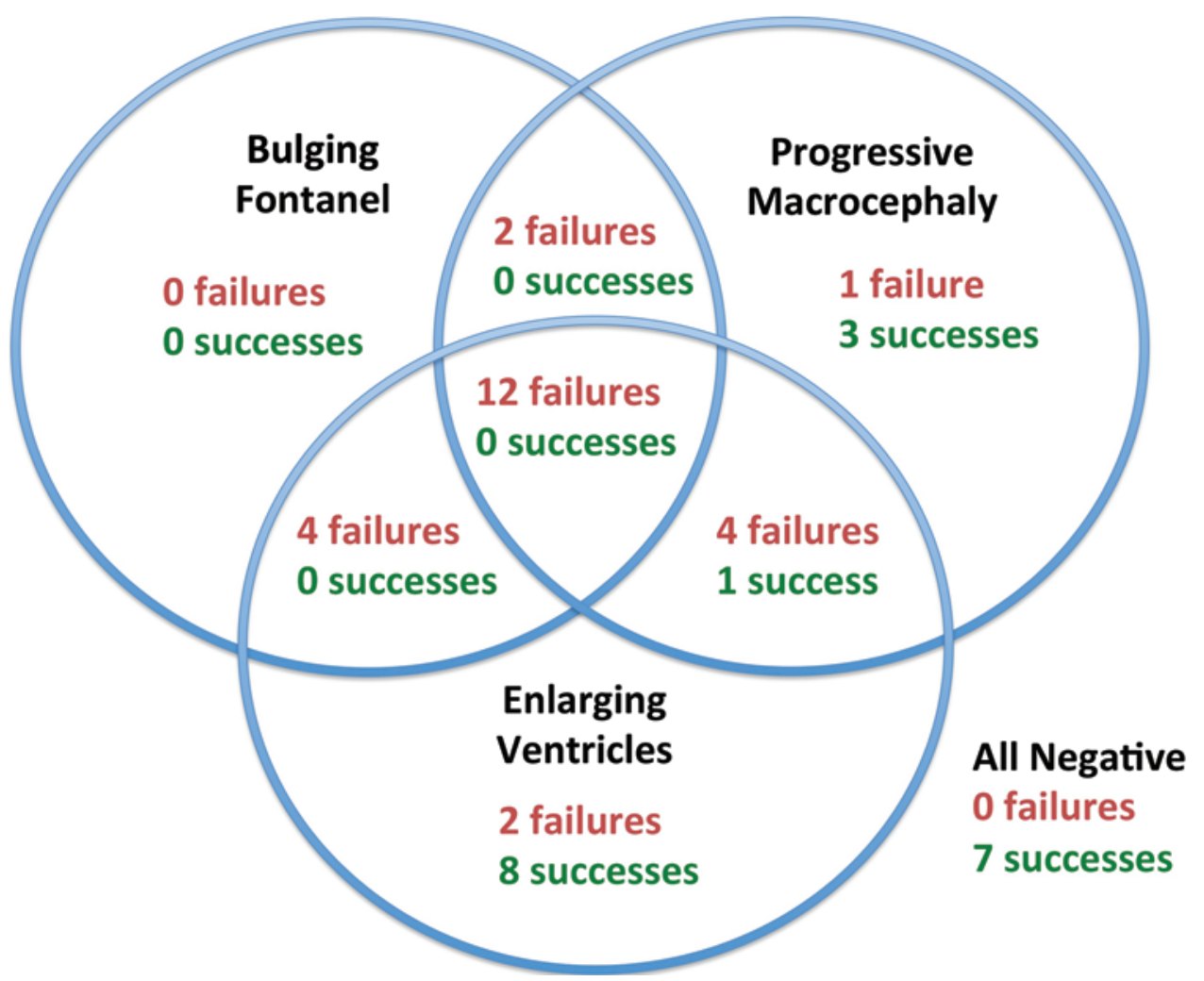

FIG. 1. Venn diagram demonstrating the interaction between hydrocephalus metrics and ETVICPC outcome. The intersection of individual circles with one another indicates the presence of positive findings (e.g., bulging fontanel and enlarging ventricles) of each respective hydrocephalus indicator. The 7 patients who demonstrated none of the 3 hydrocephalus indicators are represented in the bottom right-hand corner as "successes." Figure is available in color online only. 
$43 \%$ compares well to the success rate reported in a multicenter study of ETV/CPC success (Riva-Cambrin J: ETV/ $\mathrm{CPC}$ success and evolving indications and technique over time: an HCRN study, presentation at the Annual Meeting of the AANS/CNS Section on Pediatric Neurological Surgery, Seattle, Washington, 2015). Similar to other studies of ETV/CPC outcome, age was found to be the most important preoperative predictor of failure. ${ }^{11}$

To study decision making, postoperative hydrocephalus metrics for patients experiencing failure were compared with those experiencing success to objectively quantify variables defining ETV/CPC failure. The presence of a bulging fontanel, progressive macrocephaly (change in head circumference $\mathrm{z}$-score $>0$ ), and enlarging ventricles $(\Delta \mathrm{FOHR}>0)$ were each an independent criterion establishing treatment failure. Individually, each demonstrated a PPV of no less than $71 \%$, but fontanel status remained the most predictive indicator of ETV/CPC success or failure with a PPV of $100 \%$ and NPV of $73 \%$. As should be expected, when patients presented with combinations of these hydrocephalus metrics, the PPVs increased (when not already $100 \%$ ) and sensitivities decreased.

The diagnostic values in Tables 4 and 5 for each hydrocephalus metric display perhaps less transparent information on decision making than that presented in the Venn diagram in Fig. 1. Demonstrating the difficulty in discriminating successes from failure, this triad of hydrocephalus metrics was only abnormal in 48\% (12 patients) of failures, while all were within normal limits in only $37 \%$ (7 patients) of successes. Therefore, more often than not $(57 \%$ of all cases), patients fell into one of the other 6 areas of the Venn diagram with discordant data, rendering the diagnosis less straightforward.

Breaking this down further, successes were not homogeneous. Although 7 infants had all favorable hydrocephalus metrics, the other 12 successes had discordant clinical findings. Although, on average, ETV/CPC successes had no change in ventricle size $(\triangle \mathrm{FOHR}-0.01 \pm 0.07)$, individually, $9(47 \%)$ of the successes experienced a measurable increase in the size of the ventricles. Progressive macrocephaly was less prevalent in the patients experiencing success, with only 4 patients $(21 \%)$ having accelerated head growth. The only metric that was consistent in this cohort of successes was that no patient had a bulging fontanel.

Examining the individual ETV/CPC failures more closely reveals the same difficulty in diagnosis. Although almost half $(48 \%)$ of the failures had all 3 metrics, indicating failure, the remaining $52 \%$ of failures were diagnosed with discordant data. Ten of the remaining failures had 2 metrics indicating failure. Of these, 4 patients had a flat or concave fontanel but an increasing head circumference and increasing ventricle size. Two patients had smaller ventricles but a bulging fontanel with progressive macrocephaly. The last combination consisted of 4 patients with normal head growth but a bulging fontanel and enlarging ventricles. Interestingly, 3 patients were diagnosed as having experienced failure based on only 1 positive metric; 2 patients with increased ventricle size alone and 1 patient with progressive macrocephaly alone were diagnosed as having experienced failure. As powerful of a predictor as a bulging fontanel was found to be, it was never found in isolation. It is clear from this dissection of the individual patients that, although fontanel quality was the most powerful predictor of ETV/CPC failure, this may be related more to the temporal progression of hydrocephalus, with a bulging fontanel following an increase in ventricle size or progressive macrocephaly in some cases rather than a bulging fontanel developing first. The physiological reason for this observation is beyond the scope of this study, but it is likely influenced by a number of structural variables, including ventricular and calvarial compliance, the timing of suture synostosis, and the size and elasticity of soft tissue overlying the anterior fontanel. Lastly, it should be noted that 23 of the 44 patients had a combination of 2 or more positive hydrocephalus metrics. All but 1 infant were diagnosed with failure, yielding a PPV of having any combination of hydrocephalus metrics as $95 \%(21 / 22)$ and an NPV of $83 \%$ (sensitivity, $88 \%$; specificity, 94\%). Herein lies perhaps the most clinically useful piece of information. While external validation of these figures is needed, objective diagnostic parameters as robust as this would prove tremendously valuable for the clinical practice of any pediatric neurosurgeon managing this population.

The questions could-and should-be raised regarding which of these patients actually had failure of hydrocephalus treatment. That discussion unfortunately hinges on what failure truly is. Should the patient with a flat or concave fontanel but larger ventricles and progressive macrocephaly have been diagnosed as having experienced failure? Alternatively, should the 3 patients who only presented with a single positive metric and were diagnosed as having experienced failure been labeled as having undergone successful treatment (or at least monitored longer to determine success or failure)? The challenges of ETV/ CPC are not only in the technical nuances of the operative procedure, but rather, and equally as important, in answering these questions to distinguish successes from failures. And while we present in fine detail how this discrimination was conducted, this study is not designed to define what ETV/CPC failure truly is. This will likely remain unknown until long-term neurocognitive outcomes are available. Instead, these statistical measures of diagnostic performance (such as sensitivity and PPV) simply validate the a priori definition of failure held by the surgeon in making the clinical decision.

A fundamental challenge of accurately diagnosing ETV/CPC failure in young children arises from the fact that clinical and radiographic markers of ICP are less predictable following ETV/CPC. For hydrocephalus that is successfully shunted, ventricular volume reliably decreases, while the head circumference trajectory typically decreases or plateaus. ${ }^{8,10}$ As a reference procedure, shunting augments CSF dynamics in a completely different manner from that of endoscopic treatment, and, therefore, the same metrics used for assessment of success may change in different magnitudes. On the other hand, following a successful ETV in older children, preoperative symptoms often improve but the ventricular volume may not decrease. ${ }^{4,5}$ Additionally, infants are very different patients. In some ways, they provide more data because the head circumference is still changing relatively rapidly and the fontanel is present. The challenges arise from less reliable symptom 
interpretation/"reporting" and lack of robust experience in treating infantile hydrocephalus with methods other than shunting.

Of the 3 metrics, imaging findings added the least amount of information for our decision making. Only 2 of 25 failures were designated as failures based on imaging results alone. If a bulging fontanel is assumed to designate failure, then imaging helped discriminate success and failure in an additional 8 patients who had progressive macrocephaly without a bulging fontanel. Fontanel assessment can be done quickly, easily, and at no cost, and thus its exceptional influence is convenient. Additionally, fontanel assessment has high interrater reliability. ${ }^{18}$ This fact is especially advantageous in resource-poor settingswhence much of the ETV/CPC effort was pioneered ${ }^{12}-$ where follow-up imaging may be impractical or unavailable. Even in the developed world, this point gains merit as both health care spending limits and radiation exposure concerns discourage unnecessary imaging.

Of particular note, we were unable to identify concrete threshold values for $\triangle F O H R$ or change in head circumference z-score that accurately designated ETV/CPC failure. This is likely a result of the heterogeneous nature of hydrocephalus etiology and severity characterized by the study population. Additionally, the lack of concrete thresholds for these metrics emphasizes the value placed on the fontanel status. Nonetheless, identification of concrete parameters for $\triangle F O H R$ and change in head circumference gleaned from larger, more homogeneous cohorts might be uniquely beneficial and serve to lend greater objectivity to an often ambiguous decision tree.

Finally, hidden in the comparisons between successes and failures are important data about the ETV/CPC successes in Table 3. It should be noted that these successes differ greatly from what is commonly experienced after shunting. In general, ETV/CPC halted accelerated head growth and progressive ventriculomegaly, but head circumference and ventricle size remained relatively large. On average, successes noted only minimal decrease in the size of their ventricles, while head circumference z-score decreased by two-thirds of a point.

\section{Limitations and Future Directions}

These results describe our experience with infant hydrocephalus and how a diagnosis of ETV/CPC failure was reached at our institution. Thus, while combinations of clinical metrics of hydrocephalus are presented, it must be emphasized that their relationship with the designation of failure is directly related to-indeed, defined by-our bias as to what constitutes true ETV/CPC failure. Accordingly, the analysis above breaks down this bias into individual variables and assigns a relative value to each. We do not present this analysis in an attempt to establish diagnostic criteria of ETV/CPC success or failure or to claim that this is the correct method of diagnostics. Instead, the analysis is intended to reveal how these decisions are being made and generate motivation within the academic community to validate-or refute - these decisions. Previously, reports on ETV/CPC failure have analyzed preoperative predictors of failure or success. ${ }^{5,6,15}$ However, what is missing is an objective description of clinical success or failure. Ul- timately, the metrics described above are likely only biomarkers for successful hydrocephalus treatment, as true success is best measured with long-term cognitive and functional outcomes.

The retrospective design introduces bias-particularly treatment and observer bias. Additionally, the sample size ( $\mathrm{n}=44)$ is small, limiting more robust multivariate analyses. Lastly, the nature of ETV/CPC outcome designation poses an important limitation. A treatment failure was designated by the treating surgeon based upon the available clinical evidence (including fontanel quality, head circumference, and ventricle size). Accordingly, identifying and quantifying diagnostic criteria for ETV/CPC failure does as much to explain the surgeon's opinion about what $\mathrm{ETV} / \mathrm{CPC}$ failure is, as it does to isolate and categorize criteria for ETV/CPC failure itself.

A major theme of this paper is that success after ETV/ CPC appears to look different from success after shunt treatment. In a future study we plan to examine this concept by comparing these metrics between matched ETV/ CPC and shunt successes.

\section{Conclusions}

Discriminating ETV/CPC success from failure is often based on discordant data. After ETV/CPC, a bulging fontanel was diagnostic of ETV/CPC failure, but always present in combination with progressive macrocephaly and/or ventricular enlargement. One-quarter of failures were diagnosed in patients without a bulging fontanel. Conversely, only one-third of successes had a flat or concave fontanel, smaller ventricles, and normal head circumference growth together. More commonly, successes demonstrated either progressive macrocephaly or continued ventricular enlargement. While this study codifies hydrocephalus metrics in relation to procedural outcome, ETV/CPC success and failure continue to lack concrete definitions.

\section{Acknowledgments}

REDcap use and management is funded by Institute for Clinical and Translational Research grant support (UL1 TR000445 from NCATS/NIH).

\section{References}

1. Forfar JO, Arneil GC (eds): Textbook of Pediatrics. Edinburgh: Churchill Livingston, 1984, pp 278-330

2. Gallo P, Szathmari A, De Biasi S, Mottolese C: Endoscopic third ventriculostomy in obstructive infantile hydrocephalus: remarks about the so-called 'unsuccessful cases.' Pediatr Neurosurg 46:435-441, 2010

3. Harris PA, Taylor R, Thielke R, Payne J, Gonzalez N, Conde JG: Research electronic data capture (REDCap) - a metadata-driven methodology and workflow process for providing translational research informatics support. J Biomed Inform 42:377-381, 2009

4. Hopf NJ, Grunert P, Fries G, Resch KD, Perneczky A: Endoscopic third ventriculostomy: outcome analysis of 100 consecutive procedures. Neurosurgery 44:795-806, 1999

5. Kulkarni AV, Drake JM, Armstrong DC, Dirks PB: Imaging correlates of successful endoscopic third ventriculostomy. J Neurosurg 92:915-919, 2000

6. Kulkarni AV, Drake JM, Mallucci CL, Sgouros S, Roth J, Constantini S: Endoscopic third ventriculostomy in the treat- 
ment of childhood hydrocephalus. J Pediatr 155:254-9.e1, 2009

7. Kulkarni AV, Riva-Cambrin J, Browd SR, Drake JM, Holubkov R, Kestle J, et al: Endoscopic third ventriculostomy and choroid plexus cauterization in infants with hydrocephalus: a retrospective Hydrocephalus Clinical Research Network study. J Neurosurg Pediatr 14:224-229, 2014

8. Nowosławska E, Polis L, Kaniewska D, Mikołajczyk W, Krawczyk J, Szymański W, et al: Influence of neuroendoscopic third ventriculostomy on the size of ventricles in chronic hydrocephalus. J Child Neurol 19:579-587, 2004

9. O’Hayon BB, Drake JM, Ossip MG, Tuli S, Clarke M: Frontal and occipital horn ratio: A linear estimate of ventricular size for multiple imaging modalities in pediatric hydrocephalus. Pediatr Neurosurg 29:245-249, 1998

10. St George E, Natarajan K, Sgouros S: Changes in ventricular volume in hydrocephalic children following successful endoscopic third ventriculostomy. Childs Nerv Syst 20:834-838, 2004

11. Stone SSD, Warf BC: Combined endoscopic third ventriculostomy and choroid plexus cauterization as primary treatment for infant hydrocephalus: a prospective North American series. J Neurosurg Pediatr 14:439-446, 2014

12. Warf BC: Comparison of endoscopic third ventriculostomy alone and combined with choroid plexus cauterization in infants younger than 1 year of age: a prospective study in 550 African children. J Neurosurg 103 (6 Suppl):475-481, 2005

13. Warf BC: Endoscopic third ventriculostomy and choroid plexus cauterization for pediatric hydrocephalus. Clin Neurosurg 54:78-82, 2007

14. Warf BC, Dewan M, Mugamba J: Management of DandyWalker complex-associated infant hydrocephalus by combined endoscopic third ventriculostomy and choroid plexus cauterization. J Neurosurg Pediatr 8:377-383, 2011

15. Warf BC, Mugamba J, Kulkarni AV: Endoscopic third ven- triculostomy in the treatment of childhood hydrocephalus in Uganda: report of a scoring system that predicts success. $\mathbf{J}$ Neurosurg Pediatr 5:143-148, 2010

16. Weil AG, Fallah A, Chamiraju P, Ragheb J, Bhatia S: Endoscopic third ventriculostomy and choroid plexus cauterization with a rigid neuroendoscope in infants with hydrocephalus. J Neurosurg Pediatr 17:163-173, 2016

17. Wellons JC III, Holubkov R, Browd SR, Riva-Cambrin J, Whitehead W, Kestle J, et al: The assessment of bulging fontanel and splitting of sutures in premature infants: an interrater reliability study by the Hydrocephalus Clinical Research Network. J Neurosurg Pediatr 11:12-14, 2013

\section{Disclosures}

The authors report no conflict of interest concerning the materials or methods used in this study or the findings specified in this paper.

\section{Author Contributions}

Conception and design: Dewan, Naftel. Acquisition of data: Lim, Morgan, Gannon. Analysis and interpretation of data: Dewan, Shannon, Naftel. Drafting the article: Dewan. Critically revising the article: Dewan, Shannon, Wellons, Naftel. Reviewed submitted version of manuscript: all authors. Approved the final version of the manuscript on behalf of all authors: Dewan. Statistical analysis: Dewan, Shannon. Administrative/technical/material support: Gannon. Study supervision: Wellons, Naftel.

\section{Correspondence}

Michael C. Dewan, Department of Neurological Surgery, T-4224 Medical Center North, Nashville, TN 37232-2380. email: michael.dewan@vanderbilt.edu. 